
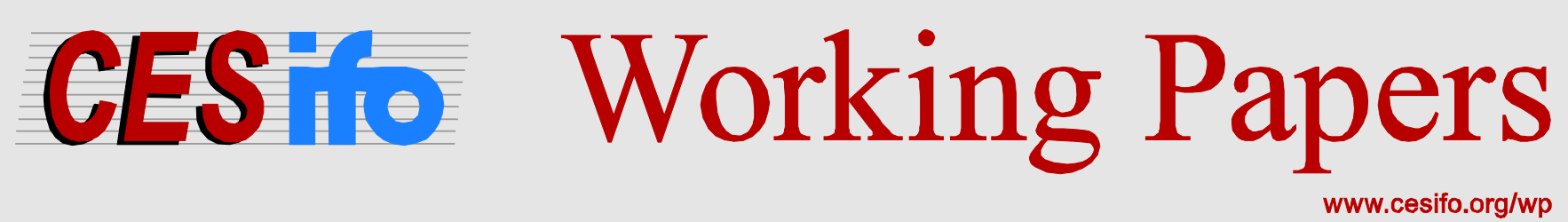

\title{
Competing Mechanisms with Limited Commitment
}

\author{
Suehyun Kwon
}

\author{
CESIFO WORKING PAPER NO. 6280 \\ CATEGORY 12: EMPIRICAL AND THEORETICAL METHODS \\ DECEMBER 2016
}

An electronic version of the paper may be downloaded

- from the SSRN website:

- from the RePEc website:

- from the CESifo website: WWW.SSRN.com

Www.RePEc.org

www.CESifo-group.org/wp

\section{CESifo}




\title{
Competing Mechanisms with Limited Commitment
}

\begin{abstract}
This paper studies competing mechanisms with limited commitment over infinite horizon. Between a mechanism and the agent, there is perfect monitoring, but each mechanism can have arbitrary signals about the interaction between other mechanisms and the agent. I show that if the agent's type is common knowledge, any individually rational payoffs can be sustained in a perfect Bayesian equilibrium. If the agent's type is his private information, Pareto frontier of mechanisms' payoffs can be obtained by repeating the static optimal screening every period; in particular, price posting with the static optimal price is the optimal mechanism. The complete information case is a strong form of folk theorem while the incomplete information case shows that folk theorem breaks down with private information even as the discount factor goes to one. Results hold with any finite number of mechanisms, any discount factor and any monitoring technology including private monitoring.
\end{abstract}

Keywords: competing mechanisms, limited commitment, private monitoring, price posting, folk theorem.

\author{
Suehyun Kwon \\ Department of Economics \\ University College London \\ 30 Gordon Street \\ United Kingdom - WC1E 6BT London \\ suehyun.kwon@ucl.ac.uk
}

December 17, 2016

This paper subsumes "Collusion with Private Monitoring and Private Information." I thank KonradvMierendorff and seminar participants at TSE, LSE and World Congress 2015. 


\section{Introduction}

Consider a firm buying intermediate goods. The interaction between a firm and a supplier is likely repeated over time, and if there are multiple sellers supplying the same intermediate good, this maps into competing mechanisms over infinite horizon. Furthermore, if suppliers cannot commit to prices they will charge in the future, there is limited commitment. I study competing mechanisms with limited commitment over infinite horizon in this paper. I characterize the set of equilibrium payoffs with complete information and Pareto frontier for mechanisms with incomplete information.

Existing literature on collusion or price competition typically models the demand side as non-strategic. Harrington and Skrypacz (2011) model the demand side as a stochastic demand function, and Athey and Bagwell (2008) also model consumers as non-strategic. Buyers in a supermarket may be anonymous, but between firms, it is more reasonable to assume that both the supplier and the buyer are long-run players. I model the buyer as a strategic long-run player, and it is one of three features that distinguish my paper from other papers. Once we model the buyer as a strategic longrun player, this naturally leads to a mechanism-design problem where each seller is a mechanism designer and the buyer is the agent. Other two features of my model are that I allow for arbitrary monitoring technology among mechanisms and I also allow for any direct mechanism. Most existing papers have focused on imperfect public monitoring, and they have also focused on price competition or auctions; literature on competing mechanisms hasn't allowed for general class of mechanisms.

However, I show that for any given Pareto weights, the weighted sum of sellers' payoffs is maximized by repeating the static optimal screening every period. No matter what is the monitoring technology among mechanisms, as long as there is perfect monitoring between each mechanism and the agent, repeating the static optimal pricing every period is the optimal mechanism. In the complete-information benchmark, any individually rational payoffs can be sustained as a perfect Bayesian equilibrium.

Since results hold for a single mechanism as well, my results provide the benchmark, mechanism with limited commitment. My results also hold for any discount factor, and it has several implications. If sellers know the buyer's valuation, any individually rational payoffs can be sustained in an equilibrium, which is stronger than the folk theorem that holds in the limit. If sellers don't know the buyer's valuation, sellers can never do better than letting the seller with the highest Pareto 
weight monopolize the market. But that seller also has to charge the static optimal price every period. Sellers can never benefit from learning the buyer's valuation over time. In addition, sellers' payoffs never converge to the Pareto frontier with complete information or the set of all individually rational payoffs. Even as the discount factor converges to 1 , the set of equilibrium payoffs with incomplete information is bounded away from the set of individually rational payoffs, and the folk theorem breaks down when the buyer has private information. My results also hold for any monitoring technology among mechanisms, including private monitoring, and they hold for both sequential equilibrium and belief-free equilibrium as well.

The rest of the paper is organized as follows. Section 2 presents the model, and results are derived in section 3 . Section 4 concludes.

\section{Model}

There are $N<\infty$ mechanisms and a single agent. Mechanisms are indexed by $i \in \mathcal{I}=\{1, \cdots, N\}$. All players share the common discount factor $\delta \in\left[\frac{1}{2}, 1\right)$ over infinite horizon, $t=1,2, \cdots 1$ Mechanisms can supply one unit of homogeneous nondurable good each period, and the agent has a unit demand each period. Mechanisms offer direct mechanisms, and the agent can participate in at most one mechanism in a given period. All mechanisms and the agent can commit only within the period.

Within each period, the payoff of mechanism $i$ from allocation $\left(q^{i}, T^{i}\right)$ is $V^{i}\left(q^{i}, T^{i}\right)=$ $T^{i} ; q^{i}$ is the probability of allocation, $T^{i} \geq 0$ is the transfer from the agent to the mechanism, and the marginal costs for mechanisms are normalized to 0 . The payoff of the agent when he participates in mechanism $i$ is $u\left(q^{i}, T^{i} \mid v\right)=q^{i} v-T^{i}$. Any mechanism that the agent doesn't participate in a given period gets 0 . If the agent doesn't participate in any mechanism, his payoff is 0 . This normalizes the outside option for all mechanisms and the agent to 0 .

In the complete information case, the agent's valuation $v$ is common knowledge, and in the incomplete information case, mechanisms start with a common prior $F$ over $[\underline{v}, \bar{v}], \underline{v} \geq q^{2}, F$ has positive density everywhere and is atomless.

I will first state the benchmark case then state the general model.

\footnotetext{
${ }^{1} \delta \geq \frac{1}{2}$ is to rule out the uninteresting case where the agent prefers to have the good this period and never again over not to have the good this period but to have it every period from the next period on.

${ }^{2}$ The upper bound on the support doesn't matter for results.
} 
In the benchmark, the agent decides which mechanism to visit, and when he visits mechanism $i$, mechanism $i$ offers a menu of contracts. The agent decides whether to participate. If the agent participates, he chooses an allocation $\left(q_{t}^{i}, T_{t}^{i}\right)$. All players receive their payoffs. The agent only sees the menu of contracts offered by the mechanism he visits, and mechanism $i$ observes the agent's participation decision and the choice of allocation; other mechanisms $j \neq i$ have no signal other than the agent didn't visit. It can be interpreted as the buyer not seeing the price until he visits the seller, and other sellers don't observe whether the buyer buys this period and from whom at what price he buys, unless the buyer comes to them.

In the general model, mechanism $i$ sends a message $m_{t}^{i} \in \mathcal{M}_{t}^{i}$ to the agent in the beginning of period $t$, and the agent decides which mechanism to visit. If the agent visits mechanism $i$, it offers a menu of contracts, and the agent decides whether to participate. If the agent participates, he chooses an allocation $\left(q_{t}^{i}, T_{t}^{i}\right)$. All players get their payoffs. Mechanism $i$ observes the agent's participation decision and the choice of allocation, and mechanisms receive $s_{t}$ distributed by $S_{t}: \Pi_{i} \mathcal{M}_{t}^{i} \times\{\{\emptyset\} \cup$ $\mathcal{I}\} \times\{\{\emptyset\} \cup \Omega\} \times\{\{\emptyset\} \cup \mathcal{A}\} \rightarrow \Delta\left(\Pi_{i} \mathcal{S}^{i}\right)$ where $\Omega$ is the set of direct mechanisms and $\mathcal{A}$ is the agent's choice of participation and allocation from a direct mechanism. $\{\emptyset\}$ correspond to the agent not visiting or not participating in any mechanism in a given period.

The general model allows mechanisms to post prices. It also allows mechanisms to post the entire menu of contracts before the agent decides which mechanism to visit. The benchmark is also an example of private monitoring, and depending on the signal structure, I can allow imperfect public monitoring, private monitoring and other variations.

In the beginning of period $t$, mechanism $i$ 's private history consists of $h^{i, t}=$ $\left(m_{1}^{i}, d_{1}^{i}, \omega_{1}^{i}, a_{1}^{i}, m_{2}^{i}, \cdots, m_{t-1}^{i}, d_{t-1}^{i}, \omega_{t-1}^{i}, a_{t-1}^{i}\right) \in \mathcal{H}^{i, t}$ where $d_{t}^{i}=1$ if the agent visits mechanism $i$ in period $t$ and 0 if he doesn't. If the agent visits, $\omega_{t}^{i}$ is the direct mechanism mechanism $i$ offers in period $t$, and $\omega_{t}^{i}=\emptyset$ if the agent doesn't visit mechanism $i$ in period $t . a_{t}^{i}$ is the allocation the agent chooses in period $t$, and if the agent doesn't visit or participate in mechanism $i$ in period $t, a_{t}^{i}=\emptyset$. After the agent decides which mechanism to visit, mechanism $i$ 's private history is $h^{i, t \prime}=$ $h^{i, t} \sqcup\left(m_{t}^{i}, d_{t}^{i}\right) \in \mathcal{H}^{i, t \prime}$. The agent's private history in the beginning of period $t$ after mechanisms sent messages is $h^{A, t}=\sqcup_{i=1}^{N} h^{i, t} \sqcup\left(m_{t}^{1}, \cdots, m_{t}^{N}\right) \in \mathcal{H}^{A, t}$. After the agent visits mechanism $i$, the agent's private history is $h^{A, t \prime}=h^{A, t} \sqcup\left(d_{t}^{1}, \cdots, d_{t}^{N}, \omega_{t}^{i}\right) \in \mathcal{H}^{A, t \prime}$. 
Strategies of mechanisms to send messages are $\sigma^{i, t}: \mathcal{H}^{i, t} \rightarrow \Delta\left(\mathcal{M}_{t}^{i}\right)$ where $\Delta(X)$ is the probability distribution over a set $X$. The strategy of the agent to visit a mechanism is $\sigma^{A, t}: \mathcal{H}^{A, t} \rightarrow \Delta(\{\emptyset\} \cup \mathcal{I})$. The strategy of mechanism $i$ when the agent visits is $\sigma^{i, t \prime}: \mathcal{H}^{i, t \prime} \rightarrow \Delta(\Omega)$, and the agent's participation and allocation strategy is $\sigma^{A, t \prime}: \mathcal{H}^{A, t \prime} \rightarrow \Delta(\{\emptyset\} \cup \mathcal{A})$. There is a public rancomization device, and the equilibrium definition is perfect Bayesian equilibrium.

\section{Results}

When mechanisms know the agent's valuation, any individually rational payoffs can be sustained as a perfect Bayesian equilibrium. However, when the agent's valuation is his private information, the weighted sum of mechanisms' payoffs can be obtained by repeating the static optimal screening every period. In particular, the mechanism with the highest weight monopolizes the market and the agent only interacts with that mechanism over infinite horizon.

The main result for the complete-information case is stated in the following theorem.

Theorem 1. Suppose the agent's valuation $v$ is common knowledge. Let $W^{i}$ be the equilibrium payoff of mechanism $i$ and $U$ is the equilibrium payoff of the agent. Any point in

$$
\left\{\left(U, W^{1}, W^{2}, \cdots, W^{N} \mid U, W^{i} \geq 0, U+\sum_{i} W^{i} \leq v\right\}\right.
$$

can be sustained in a perfect Bayesian equilibrium.

Proof. The proof is by construction. Let $\omega^{j} \in\left\{M_{1}, M_{2}, A, M C, N T, T\right\}$ be the state variable for Markov strategies of mechanism $j$ and the agent. The first three are the indicator function of who deviated the last between mechanism $j$ and the agent. If the agent deviated the last, $\omega^{j}=A$; if mechanism $j$ sent an off-the-equilibrium path message, $\omega^{j}=M_{1}$, and if it offered an off-the-equilibrium path contract, $\omega^{j}=M_{2}$. $M C, T, N T$ indicate whether they are in the marginal-cost pricing regime, the trade regime or the no-trade regime. Consider the following strategies. While $\omega^{j}=M C$,

1. mechanism $j$ sends no message to the agent, 
2. the agent visits mechanism $j$,

3. mechanism $j$ offers $\left(q_{t}^{j}, T_{t}^{j}\right)=(1,0)$,

4. the agent participates and takes $\left(q_{t}^{j}, T_{t}^{j}\right)=(1,0)$.

While $\omega^{j}=N T$,

1. mechanism $j$ sends no message to the agent,

2. the agent doesn't visit mechanism $j$.

While $\omega^{j}=T$,

1. mechanism $j$ sends no message to the agent,

2. the agent visits mechanism $j$,

3. mechanism $j$ offers $\left(q_{t}^{j}, T_{t}^{j}\right)=(1, v-U)$,

4. the agent participates and takes $\left(q_{t}^{j}, T_{t}^{j}\right)=(1, v-U)$.

When $\omega^{j}=M_{1}$,

1. the agent doesn't visit,

2. in the following period, $\omega^{j}=N T$.

When $\omega^{j}=M_{2}$,

1. the agent doesn't participate this period,

2. in the following period, $\omega^{j}=M C$.

When $\omega^{j}=A$,

1. if the last deviation is that (i) the agent didn't visit when his strategy was to visit, (ii) the agent didn't participate when his strategy was to participate, or (iii) if the last deviation is that the agent participated when his strategy was not to participate, $\omega^{j}=N T$ in the following period.

2. if the last deviation is that the agent visited when his strategy was not to visit,

(a) mechanism $j$ offers $\left(q_{t}^{j}, T_{t}^{j}\right)=(0, v)$, 
(b) the agent doesn't participate,

(c) $\omega^{j}=N T$ in the following period.

Given $\left(U, W^{1}, \cdots, W^{N}\right)$, there exist $\mu_{1}, \cdots, \mu_{N} \in[0,1]$ such that $W^{i}=\mu_{i}(v-$ $U), \forall i$ and $\sum \mu_{i} \leq 1$. In the first period, with probability $\mu_{i}, \omega^{i}=T$ and $\omega^{j}=N T$ for all $j \neq i$. The rest is to verify that the strategies above define an equilibrium. Since we have continuity at infinity, it is sufficient to check that no player has incentives to deviate when there has been a finite number of deviations across all players.

After any history, there is at most one mechanism $i$ where $\omega^{i}=T$ or $M C$. All other mechanisms have one of $\omega^{j}=A, M_{1}, M_{2}$ or $N T$. Once $\omega^{i}$ leaves $T$, it never goes back to $T$ and has to be one of $A, M_{1}, M_{2}, M C, N T$. In the first period, there is at most one mechanism with $\omega^{i}=T$, and therefore, there can never be more than one mechanism with $\omega^{i}=T$. In order to go to $\omega^{j}=M C$, the agent has to visit mechanism $j$ and the mechanism $j$ has to offer an off-the-equilibrium path contract. Since the agent can only visit one mechanism in a given period, there can be at most one mechanism with $\omega^{j}=M C$. The last step is to show that there can't be two mechanisms such that $\omega^{i}=T, \omega^{j}=M C, i \neq j$. But both $T$ and $M C$ require the agent to visit their mechanisms, and this can't happen simultaneously.

From any mechanism's perspective, when it initiates the deviation and if it's the last deviation, $\omega^{j}$ becomes $M_{1}$ then $N T$ or $M_{2}$ then $M C$ regardless of the current $\omega^{j}$. It's deviation payoff is 0 , and the mechanisms don't have incentives to deviate.

We just need to verify the agent's incentives to deviate. The agent's deviation payoff is 0 possibly except when the agent wasn't supposed to participate but he participates, and this is the only case we need to verify. If the mechanism offers an off-the-equilibrium path contract and the agent's participation is the last deviation, the agent's deviation payoff is at most $(1-\delta) v$. His continuation value from not participating is $\delta v$ by the marginal-cost pricing regime, and $\delta \geq \frac{1}{2}$ implies that the agent doesn't deviate. If mechanisms follow their strategies, there is one more subcase to check. If the agent's strategy was to visit mechanism $i$, his strategy is to participate in mechanism $i$ for all $\omega^{i} \neq M_{2}$. Therefore, the agent's strategy must have been not to visit mechanism $i$ but he visited and he also participated even though his strategy is not to participate. But if mechanism $i$ didn't deviate after the agent visited, it offers $(0, v)$, and by participating, the agent's deviation payoff is $-(1-\delta) v$. Since by not participating, the agent can guarantee at least 0 , the agent doesn't participate. 
Therefore, the above strategies form an equilibrium.

In this equilibrium, the strategies of mechanism $i$ and the agent only depend on the interaction between the two, and there is perfect monitoring between each mechanism and the agent. Therefore, when the other player is playing a Markov strategy only conditional on the private history between the two, it is an equilibrium to ignore any signal about the interaction between other mechanisms and the agent. Also, in this equilibrium, mechanisms don't send messages, but the proof can be adapted so that the mechanisms post the menu of contracts. If a mechanisms posts a menu of contracts in its message but offers a different menu of contracts when the agent visits, it triggers $\omega^{i}$ to become $M_{2}$, and the rest of the equilibrium construction works the same way.

I used the public randomization device so that with probability $\mu_{i}, \omega^{i}=T$ and $\omega^{j}=N T$ for all $j \neq i$ in the first period, but we can modify the proof so that there is no public randomization device. This would require the strategies to be defined as above from the second period onwards, and in the first period the agent randomizes among all mechanisms. Since all mechanisms follow the same strategy, the agent is indifferent across all of them and is willing to randomize.

The next theorem characterizes Pareto frontier for mechanisms when the agent's valuation is his private information.

Theorem 2. Suppose the agent's valuation is his private information. Pareto frontier of mechanisms' payoffs is given by

$$
\left\{\left(W^{1}, \cdots, W^{N}\right) \mid W^{i}, \mu_{i} \geq 0, \forall i, \sum \mu_{i}=1, \sum \mu_{i} W^{i}=(1-F(\hat{v})) \hat{v}\right\}
$$

If the solution to

$$
v=\frac{1-F(v)}{f(v)}
$$

is in the support, $\hat{v}$ is the solution to the above equation. If not, $\hat{v}=\underline{v}$. The buyer's payoff is $v-\hat{v}$ if $v \geq \hat{v}, 0$ otherwise.

Proof. Suppose given Pareto weights $\left(\mu_{1}, \cdots, \mu_{N}\right), \lambda_{v}$ and $T_{v}$ are the discounted weighted sum of the probabilities type $v$ buys from any of the sellers and the dis- 
counted sum of the payments he makes:

$$
\begin{aligned}
& \lambda_{v}=\sum_{t=1}^{\infty} \delta^{t-1} \sum_{i=1}^{N} \mu_{i} \lambda_{v}^{i, t} \\
& T_{v}=\sum_{t=1}^{\infty} \delta^{t-1} \sum_{i=1}^{N} \mu_{i} T_{v}^{i, t}
\end{aligned}
$$

where $\lambda_{v}^{i, t}$ is the ex ante probability that type $v$ buys from mechanism $i$ in period $t$ and $T_{v}^{i, t}$ is the ex ante payment type $v$ makes to mechanism $i$ in period $t$.

One of the IC constraints is that type $v$ and $v^{\prime}$ don't mimic each other forever:

$$
\begin{gathered}
\lambda_{v} v-T_{v} \geq \lambda_{v^{\prime}} v-T_{v^{\prime}}, \\
\lambda_{v^{\prime}} v^{\prime}-T_{v^{\prime}} \geq \lambda_{v} v^{\prime}-T_{v} .
\end{gathered}
$$

We can combine the two IC constraints as $\left(\lambda_{v}-\lambda_{v^{\prime}}\right) v \geq T_{v}-T_{v^{\prime}} \geq\left(\lambda_{v}-\lambda_{v^{\prime}}\right) v^{\prime}$, and the local ICs are sufficient:

$$
\lim _{v^{\prime} \rightarrow v}\left(\lambda_{v}-\lambda_{v^{\prime}}\right) v=T_{v}-T_{v^{\prime}}
$$

The relaxed problem with IC constraints that type $v$ doesn't mimic $v^{\prime}$ forever is as follows:

$$
\begin{gathered}
\max \int T_{v} d F(v) \\
\text { s.t. } \lambda_{v} \in[0,1] \forall v, \\
I C: \lim _{v^{\prime} \rightarrow v}\left(\lambda_{v}-\lambda_{v^{\prime}}\right) v=T_{v}-T_{v^{\prime}}, \\
I R: \lambda_{\underline{v}} \underline{v}-T_{\underline{v}}=0 .
\end{gathered}
$$

From the IC constraint, we get

$$
\begin{aligned}
\int T_{v} d F(v) & =\int\left(v \lambda_{v}-\int_{\underline{v}}^{v} \lambda_{z} d z\right) d F(v) \\
& =\int\left(v \lambda_{v}-\frac{1-F(v)}{f(v)} \lambda_{v}\right) d F(v) .
\end{aligned}
$$

Since $\lambda_{v}$ is bounded, we get 0 or 1 as the solution, and from the monotone hazard 
rate condition, there exists $\hat{v}$ such that $\lambda_{v}=1$ if and only if $v \geq \hat{v} . \lambda_{v}=0$ otherwise.

The next step is to show that there exists a perfect Bayesian equilibrium of the full problem with the same property. Let $i$ be the mechanism with the highest Pareto weight $\mu_{i}$. (if there are multiple mechanisms with the same Pareto weight, pick the minimum $i$ ) Consider the equilibrium from the proof of Theorem 1 where the transfer $v-U$ for $\omega^{j}=T$ is replaced by $\hat{v}$ and the transfer $v$ for $\omega^{j}=A$ is replaced by $\bar{v}$.

In the first period, mechanisms don't send messages, and the agent visits mechanism $i$ if $v \geq \hat{v}$ and doesn't visit any mechanism if $v<\hat{v}$. All mechanisms $j \neq i$ has $\omega^{j}=N T$, and mechanism $i$ has $\omega^{i}=T$ if the agent visits and $\omega^{i}=N T$ if the agent doesn't visit. The rest is to verify that these strategies form an equilibrium, but since the strategies are determined by the interaction between each mechanism and the agent, we just need to verify that the agent has the right incentives in the first period. If the agent visits mechanism $i, \omega^{i}$ becomes $T$, and the agent gets $v-\hat{v}$ over infinite horizon. If the agent doesn't visit mechanism $i, \omega^{i}$ becomes $N T$; if the agent visits some other mechanism $j, \omega^{j}=A$, and otherwise all mechanisms have $\omega^{k}=N T$. Once $\omega^{k}$ 's are determined in the first period, it is an equilibrium to follow the strategies. If the only deviation is whether the agent visits any mechanism and which mechanism he visits, the agent compares $v-\hat{v}$ and 0 , and it is the best response of the agent to visit mechanism $i$ in the first period if and only if $v \geq \hat{v}$.

When the agent's valuation is his private information, Pareto frontier for mechanisms is obtained by repeating the static optimal screening every period. The mechanism with the highest Pareto weight monopolizes the market, and the agent visits this mechanism every period if his valuation is above the cutoff. The agent never visits any mechanism if his valuation is below the cutoff.

The proof consists of two steps. I first put an upper bound on any weighted sum of mechanisms' payoffs then show that the upper bound can be attained as an equilibrium. The second step of constructing the equilibrium follows the proof of Theorem 1 closely. In the first step, I derive the upper bound by consider a relaxed problem. In principle, when we allow arbitrary signals about interaction between other mechanisms and the agent and when we also allow any mechanism, the set of IC constraints is very big. When I consider the relaxed problem, I ignore mechanisms' incentives altogether, and also for the agent, I only consider the deviation strategy of mimicking another type forever. When we only consider the IC constraint of the agent mimicking another type forever, maximizing the weighted sum of mechanisms' 
payoffs maps into a static mechanism design problem, and the optimal mechanism is to sell with probability 1 if the virtual surplus is positive and never sell if the virtual surplus is 0 . Since adding additional constraints only weakly lower the weighted sum of mechanisms' payoffs, constructing an equilibrium with the maximum payoff from the relaxed problem shows that this is the tight upper bound. I also allow for any Pareto weights, and the equilibrium construction traces all of Pareto frontier for mechanisms.

Theorems 1 and 2 have several implications which are discussed in the next section.

\section{Conclusion}

I studied competing mechanisms with limited commitment over infinite horizon in this paper. I characterized the set of equilibrium payoffs when the agent's valuation is common knowledge among mechanisms and Pareto frontier for mechanisms when the agent's valuation is his private information.

First of all, Theorem 1 shows that if the agent's valuation is common knowledge among mechanisms, any individually rational payoffs can be sustained as an equilibrium with competing mechanisms and limited commitment. Theorem 1 holds with any discount factor bounded away from one, and it is stronger than folk theorem that holds in the limit.

Second, Theorem 2 shows that folk theorem result with complete information completely breaks down when the agent has private information about his valuation. Mechanisms can never do better than repeating the static optimal screening every period, and in particular, even as the discount factor converges to one, the set of equilibrium payoffs never converge to the set of individually rational payoffs which is the same as the set of equilibrium payoffs with complete information.

Third, both theorems essentially work with sequential equilibrium or belief-free equilibrium as well. Since the state variables for Markov strategies only depend on the private history between each mechanism and the agent and there is perfect monitoring between each pair, folk theorem with complete information and anti-folk theorem with incomplete information work with those equilibrium definitions as well.

Fourth, Theorem 2 also shows that learning over time doesn't help mechanisms. Mechanism $i$, for example, learns whether the agent's valuation is above or below $\hat{v}$ in the first period, but it can't leverage that information. Literature on ratchet effect 
suggests that mechanisms might learn over time and exploit the agent, but there is discontinuity between finite-horizon models and infinite-horizon models, and with infinite horizon, there is always a continuation game, and one can always construct a no-trade equilibrium or marginal-cost pricing equilibrium as continuation strategies.

Fifth, Theorems 1 and 2 show that modelling the buyer (the agent) as a strategic long-run player has a significant impact on the equilibrium payoffs of the sellers (mechanisms).

Sixth, the combination of perfect monitoring between each mechanism and the agent and Markov strategies only using the private history between each pair shows that once it is an equilibrium to follow the Markov strategies between the pair, there is really nothing other mechanisms can do to deter the pair or to attract the agent to come visit them instead. This is why any signal structure about the interaction between other mechanisms and the agent can be allowed for yet it makes no difference to the set of equilibrium payoffs.

\section{References}

[1] Athey, Susan, and Kyle Bagwell. "Optimal collusion with private information." RAND Journal of Economics 32 (2001): 428-465.

[2] Athey, Susan, and Kyle Bagwell. "Collusion with Persistent Cost Shocks." Econometrica 76 (2008): 493-540.

[3] Bergemann, Dirk, and Juuso Välimäki. "Information acquisition and efficient mechanism design." Econometrica 70 (2002): 1007-1033.

[4] Bergemann, Dirk, and Juuso Välimäki. "Dynamic price competition." Journal of Economic Theory 127 (2006): 232-263.

[5] Green, Edward J., and Robert H. Porter. "Noncooperative collusion under imperfect price information." Econometrica 52 (1984): 87-100.

[6] Harrington, Joseph E., and Andrzej Skrzypacz. "Collusion under monitoring of sales." RAND Journal of Economics 38 (2007): 314-331. 
[7] Harrington, Joseph E., and Andrzej Skrzypacz. "Private monitoring and communication in cartels: Explaining recent collusive practices." American Economic Review 101 (2011): 2425-2449.

[8] Stigler, George J. "A theory of oligopoly." Journal of Political Economy 72 (1964): 44-61. 